\title{
Lumbar Decompressive Laminectomy or Laminotomy for Degenerative Conditions: "Outcome Comparison of Traditional Open versus Less Invasive Techniques"
}

Reginald Q Knight ${ }^{1,4}$, Melissa Scribani ${ }^{2}$, Nicole Krupa $^{2}$, Scott Grainger ${ }^{1}$, Craig Goldberg ${ }^{3}$, Carl Spivak ${ }^{1,4}$ and Paul Jenkins $^{2}$

${ }^{1}$ Bassett Spine Care Institute, Cooperstown, New York, USA

${ }^{2}$ Bassett Research Institute, Cooperstown, New York, USA

${ }^{3}$ St. Peters Hospital, Latham, New York, USA

${ }^{4}$ Columbia University College of Physicians and Surgeons, New York, USA

\section{Abstract}

Study design: Non-randomized chart review of elective lumbar decompression

Objective: Compare patient outcome and health system economic impact associated with direct lumbar decompression.

Summary of background data: Degenerative lumbar conditions refractory to non-operative measures are traditionally treated via open decompression. Less invasive techniques assisted by tubular retractors or endoscopic visualization continue to grow in popularity.

Methods: 338 consecutive patients with spinal stenosis or disc herniation were treated with: Open, Tube-assisted, or Endoscope-assisted procedures based on the surgeons' typical indications, practice pattern and procedure of choice. Cases stratified by stenosis requiring decompression without discectomy (Stenosis) or disc herniation requiring discectomy (Disc). Data collected preoperatively, one, four and ten months postoperatively. Within strata, perioperative demographics, intraoperative and postoperative complications, and functional outcomes were compared across procedure types. Outcome measures include VAS (back / leg), Oswestry and Medicare subset for Net revenue.

Results: 234 Disc and 104 Stenosis cases. Stenosis patients were significantly older than Disc patients $(67.0$ vs. 52.3 years, $p=0.0001$ ). Disc cases: $42.7 \%$ Open, $36.8 \%$ Endoscope-assisted, $20.5 \%$ Tube-assisted. Stenosis cases: $36.5 \%$ Open, $63.5 \%$ Tube-assisted. Operative time, estimated blood loss, and length of stay were significantly greater for Open procedures both Disc and Stenosis. Disc cases fluoroscopy time was significantly greater for Endo $(p<0.0001)$. Stenosis cases fluoroscopy time was significantly greater for Tube-assisted.

Intraoperative complications occurred in 12 (3.5\%) patients, 16 experienced postoperative events. A non-significant trend towards greater post-operative complication was seen in Open - stenosis group.

Functional outcome improvements for ODI, VASB, and VASL were experienced regardless of case group or procedure type $(p<0.0001)$. Medicare $(n=107)$ revenue generated net positive regardless of case type or location.

Conclusions: Functional improvement following treatment of degenerative lumbar conditions via direct decompression should be anticipated regardless of case group or procedure type. Despite their reduction in fluoroscopy, Open cases are associated with a significant increase in length of stay, operating time, estimated blood loss and potentially postoperative infections.

Keywords: Discectomy; Blood loss; Microendoscopic; Stenosis patient

\section{Keypoints}

- Direct decompression of lumbar degenerative conditions should be associated with improved patient functional outcome.

- Less invasive techniques such as endoscopic or tubular decompression are associated with significant reduction in length of stay, operating time, estimated blood loss and potentially postoperative infections when compared to open techniques.

- In-patient surgical services for degenerative conditions requiring direct decompression are economically advantageous in comparison to outpatient locations in a Medicare population.

\section{Introduction}

Lumbar degenerative conditions requiring medical evaluation affect a growing number of the population [1]. Traditional treatment of refractory symptoms is comprised of open direct decompression via laminectomy or laminotomy. Recently smaller incisions and reduced bone resection have gained popularity [2,3]. Procedures utilizing modifications of standard retractors, development of new instrumentation systems, and adaption of non-traditional approaches continue to evolve.

Minimally invasive surgery utilizing tubular retractors and endoscopes have captured imagination of patients and surgeons [1,47]. Reduced morbidity is a common finding when comparing open and minimally invasive procedures for lumbar decompression [1,6-10]. Shih et al. demonstrated reductions in hospital stay, estimated blood loss and need for ancillary services associated with microendoscopic care [6].

*Corresponding author: Reginald Q Knight, Bassett Spine Care Institute Division of Orthopedic Surgery, Bassett Healthcare Network, One Atwell Road Cooperstown, New York 13326, USA, Tel: (607) 547-3456; Fax: (607) 547-4067 E-mail: Reginald.knight@bassett.org

Received October 18, 2013; Accepted October 22, 2013; Published October 25, 2013

Citation: Knight RQ, Scribani M, Krupa N, Grainger S, Goldberg C, et al.(2013) Lumbar Decompressive Laminectomy or Laminotomy for Degenerative Conditions: "Outcome Comparison of Traditional Open versus Less Invasive Techniques". J Spine S2: 006. doi:10.4172/2165-7939.S2-006

Copyright: ( 2013 Knight RQ, et al. This is an open-access article distributed under the terms of the Creative Commons Attribution License, which permits unrestricted use, distribution, and reproduction in any medium, provided the original author and source are credited. 
Despite reported reductions in blood loss, narcotic use and length of hospital stay utilizing minimally invasive techniques, researchers have also documented increases in operative time $[6,10]$ or patient reported back and leg pain [4] attributed to newer techniques.

Medical or surgical intervention's primary goal is improvement in patient functional outcome. Measuring functional improvement is accomplished using multiple standardized instruments - Short-form 36, Oswestry Disability Index (ODI), Visual Analog Scales (VASB and VASL), Roland-Morris Disability Questionnaire and others. Parker and colleagues demonstrated positive correlation between minimally invasive lumbar decompression and overall quality of life [11,12]. Procedure type, open or minimally invasive, does not appear to negatively impact this expectation $[4,7,10,11,13]$.

In today's recessionary environment, healthcare economics are of growing importance. Healthcare systems face increasing accountability for services provided. Improvements in quality of life and cost-effectiveness related to lumbar decompression have been established [11,12]. Medicare, through DRG and APC programs, has established reimbursement formulas based on regional conversion factors and presence or absence of patient co-morbidities [14]. Lumbar decompression is traditionally provided through inpatient settings. Growing use of ambulatory centers has drawn attention from regulatory agencies. Improved patient function following direct lumbar decompression remains a standard. How are providers, patients and payer likely to guide their decision regarding procedural choice-open versus minimally invasive or location - inpatient versus outpatient?

Our research goal is documentation of perioperative differences in morbidity associated with open versus minimally invasive decompression of lumbar spine for degenerative conditions. Secondarily, and perhaps more importantly, we summarize economic impact associated with choice of inpatient or outpatient setting in our Medicare population.

\section{Methods}

Our system is a rural multispecialty employed physician model covering nine counties of Central New York State. In January 2010, we initiated a prospective registry for all operative spine care. Participation is voluntary. Functional outcome data and chart review for perioperative demographics was compiled on 338 consecutive elective lumbar degenerative cases. Patients with symptoms of neurogenic claudication or lumbar radiculopathy refractory to organized non-operative care were considered candidates for study. Patients were excluded for: osteoporosis, fracture, tumor, spondylolis thesis or spinal instability requiring fusion. Surgeons selected procedures based on clinical judgment and patient presentation.

Cases were categorized by procedure type: Open, Tube or Endoscope-assisted direct neural decompression included laminectomy, laminotomy and for aminotomy at clinically relevant levels. The degree of lumbar stenosis on MRI or CT scan was not reported as part of this study. Levels indicated for decompression or discectomy were based on the surgeon's interpretation of clinical findings - history, physical examination and imaging studies (Table 2). Surgeons selected procedure type based on their own practice pattern. Four surgeons participated in this study and there was no alteration in procedure choice based on their indication for surgery. Open procedures (performed by two surgeons) and tube-assisted procedures (performed by one surgeon) were performed bilaterally or unilaterally according to surgeons discretion based on patient pathology. Endoscope-assisted procedures (performed by one surgeon) were performed unilaterally. Cases were categorized as Disc or Stenosis patients. Disc patients presented with primary symptoms of radiculopathy consistent with imagining studies and required discectomy of herniated tissue. Stenosis patients presented with primary symptoms of neurogenic claudication and imagining studies illustrating clinically consistent lumbar canal stenosis-central, lateral or foraminal.

Functional outcome data, Oswestry Disability Index (ODI), Visual Analog Scale (0-100) for back (VASB) and leg (VASL) pain was collected preoperatively, at one, four, and ten months postoperatively. Perioperative demographics collected include: hospital stay (days), estimated blood loss (cc's), operative time (minutes), fluoroscopy time (seconds), levels decompressed, occurrence of intraoperative or postoperative complications, and discharge status.

Hospital finance data from January 2012 through July 2012 generated a generic Med/Surgery cost per day of hospital stay ( $\$ 1,150$, excluding dietary and maintenance cost) and cost per minute of operating room time ( $\$ 22.10$ per minute). Using Medicare Diagnosis-Related Groups (DRGs) and Ambulatory Payment Classification (APC) data, a subset of 107 Medicare patients (32\% of Total Cohort) underwent financial analysis. DRG 490 (with co-morbidities) was selected in relationship to Current Procedural Terminology (CPT) 63047 or 63048. Our finance group data using National Case Mix Index (1.7987) resulted in reimbursements of $\$ 15,820$ (hospital) and $\$ 1,017$ (professional) based on CPT. Our regional APC reimbursements $\$ 3,501$ (facility) and \$1,017 (professional). Mean operative times (outpatient and inpatient) and length of stay for this subset of Medicare patients was calculated (Table 6).

\section{Data analysis}

Demographics were compared between Disc and Stenosis patients. Proportions of males and females were compared using two-by-two chi-square, and mean age was compared using t-test. Distribution of insurance types was compared using two-by-five chi-square.

Analyses comparing procedure type was stratified by Disc/Stenosis. Within each stratum, proportion of males/females was compared across procedure types (Open, Endo, Tube) using two-by-three chi-square. Mean age was compared across procedure types using one-by-three analysis of variance (ANOVA) where nature of any significant main effects was explored using Scheffe's pair-wise post hoc comparison test. Continuous perioperative variables were compared across procedure types using one-way ANOVA and Scheffe's post-hoc comparisons. Proportions of intraoperative and postoperative complications and of patients discharged to skilled nursing facilities were compared between procedure types using Fisher's Exact test. Median cost of Medicare inpatient procedures was compared across procedure types using Kruskal-Wallis test. Functional improvements (ODI, VASB, VASL) were compared over time and between procedures using two-way ANOVA. All analyses were conducted using SAS 9.1 (Cary, NC) and p-values $\leq 0.05$ were considered statistically significant. Research was conducted under the auspices of ongoing IRB review.

\section{Results}

\section{Cohort demographics (Table 1)}

There are 338 consecutive patients studied, 192 male (56.8\%) 146 female (43.2\%). The Stenosis group was $66.0 \%$ male, and Disc group $52.6 \%$ male. Patients in Stenosis group were significantly older than Disc group (67.0 years vs. 52.3 years, respectively, $\mathrm{p}<0.0001$ ).

Open procedures were most commonly used, 138 (40.8\%) patients undergoing this procedure type. However, when considering Stenosis 
Citation: Knight RQ, Scribani M, Krupa N, Grainger S, Goldberg C, et al.(2013) Lumbar Decompressive Laminectomy or Laminotomy for Degenerative Conditions: "Outcome Comparison of Traditional Open versus Less Invasive Techniques". J Spine S2: 006. doi:10.4172/2165-7939.S2-006

Page 3 of 10

\begin{tabular}{|c|c|c|c|c|}
\hline Category & Total Cohort & Stenosis Group & Disc Group & p (disc vs. stenosis \\
\hline Total Subjects & 338 & $104(30.8 \%)$ & $234(69.2 \%)$ & \\
\hline Male & $192(56.8 \%)$ & $69(66.0 \%)$ & $123(52.6 \%)$ & 0.02 \\
\hline Female & $146(43.2 \%)$ & $35(34.0 \%)$ & $111(47.4 \%)$ & \\
\hline Endoscope-assisted & $86(25.4 \%)$ & -- & $86(36.8 \%)$ & \\
\hline Open & $138(40.8 \%)$ & $38(36.5 \%)$ & $100(42.7 \%)$ & \\
\hline Tube-assisted & $114(33.7 \%)$ & $66(63.5 \%)$ & $48(20.5 \%)$ & \\
\hline Mean age (SD) & $56.9(15.9)$ & $67.0(13.2)$ & $52.3(14.9)$ & $<0.0001$ \\
\hline Private Insurance & $164(48.2 \%)$ & $35(33.7 \%)$ & $129(55.1 \%)$ & $<0.0001$ \\
\hline Medicare & $107(32.1 \%)$ & $56(53.9 \%)$ & $51(21.8 \%)$ & \\
\hline Worker's Compensation & $31(9.1 \%)$ & $8(7.7 \%)$ & $23(9.8 \%)$ & \\
\hline Medicaid & $30(8.8 \%)$ & $5(4.8 \%)$ & $25(10.7 \%)$ & \\
\hline Uninsured & $6(1.8 \%)$ & 0 & $6(2.6 \%)$ & \\
\hline
\end{tabular}

Table 1: Cohort Demographics.

\begin{tabular}{|c|c|c|c|c|c|}
\hline & \multirow[t]{2}{*}{ Total Cohort } & \multicolumn{2}{|c|}{ Stenosis Group ( $n=104)$} & \multicolumn{2}{|c|}{ Disc Group $(n=234)$} \\
\hline & & Mean & p-value (method) & Mean & $p$-value (method) \\
\hline OR Time (Min) & 121.1 & 151.2 & & 107.7 & \\
\hline Endo & 86.4 & -- & & 86.4 & \\
\hline Open & 143.6 & 194.8 & $<0.0001$ & 124.1 & $<0.0001$ \\
\hline Tube & 120.1 & 126.1 & & 111.8 & \\
\hline EBL (cc's) & 68.0 & 119.9 & & 45.0 & \\
\hline Endo & 25.0 & -- & & 25.0 & \\
\hline Open & 119.1 & 250.3 & $<0.0001$ & 69.2 & 0.0004 \\
\hline Tube & 38.7 & 44.9 & & 30.3 & \\
\hline Fluoroscopy Time (sec) & 33.7 & 12.9 & & 43.7 & \\
\hline Endo & 87.1 & -- & & 87.1 & \\
\hline Open & 2.7 & 0.2 & $<0.0001$ & 3.8 & $<0.0001$ \\
\hline Tube & 24.6 & 19.4 & & 31.8 & \\
\hline LOS & 1.1 & 1.7 & & 0.9 & \\
\hline Endo & 0.1 & -- & & 0.1 & \\
\hline Open & 1.9 & 3.4 & 0.002 & 1.4 & $<0.0001$ \\
\hline Tube & 0.9 & 0.7 & & 1.1 & \\
\hline Levels Decompressed & 1.3 & 1.7 & & 1.1 & \\
\hline Endo & 1.0 & -- & & 1.0 & \\
\hline Open & 1.3 & 1.9 & 0.11 & 1.1 & 0.20 \\
\hline Tube & 1.4 & 1.6 & & 1.1 & \\
\hline
\end{tabular}

$\mathrm{OR}=$ Operating Room, EBL = Estimated Blood Loss, Endo = Endoscope-assisted, Sign = significance

Tube $=$ Tube-assisted, $\sec =$ seconds

Min= minutes

Table 2: Perioperative Demographics

patients, Tube-assisted procedures were used more often (Tube 63.5\%, Open $36.5 \%)$.

Nearly half (48.2\%) of all patients had private insurance coverage, while about one-third (32.1\%) were Medicare-covered. Only $1.8 \%$ of all patients were uninsured. Distribution of insurance types differed significantly between Stenosis and Disc groups. This relationship was primarily driven by higher percentage of Medicare patients in Stenosis group (53.9\%) vs. Disc group (21.8\%), $\mathrm{p}<0.0001)$.

\section{Perioperative demographics (Table 2)}

Overall mean length of stay was 1.1 days for all patients. Among 
Stenosis patients, Open procedures produced significantly longer LOS (3.4 days) compared to Tube-assisted procedures ( 0.7 days, $\mathrm{p}=0.002)$. Similarly, Disc patients with Open procedures experienced longer LOS (1.4 days) compared to Tube-assisted (1.1 days) or Endoscopic (0.1 days).

Open procedures were associated with longer mean operative times in both Stenosis and Disc patients. Among Stenosis patients, Open procedures took an average of 194.8 minutes, as compared to 126.1 minutes $(\mathrm{p}<0.0001)$ for Tube. Disc patients undergoing Open procedures spent an average of 124.1 minutes in the OR, as compared Endo and Tube procedures (111.8 and 86.4 minutes, respectively, $\mathrm{p}<0.0001)$.

Estimated blood loss for Open procedures was also significantly greater for both Stenosis and Disc patients. One intraoperative blood transfusion was administered (Stenosis patient undergoing Open procedure).

There was no significant difference in number of levels decompressed between Open and Tube-assisted for Stenotic conditions. Similarly, no difference in levels decompressed was observed across three procedure types among Disc patients. No attempt was made to stratify data regarding unilateral versus bilateral decompression. Decompression was pathology appropriate as dictated by the physician.
Among Stenosis patients, fluoroscopy time in seconds was significantly greater for Tube procedures as compared to Open procedures $(\mathrm{p}<0.0001)$. Among Disc patients, fluoroscopy time was significantly greater for Endoscopic procedures as compared to Open or Tube $(\mathrm{p}<0.0001)$.

\section{Complications and discharge status (Table $3 a$ and $3 b$ )}

Intraoperative complications occurred in 12 patients (3.5\%; 1 Endo, 7 Open, 4 Tube cases). Dural tears accounted for 11 (91.7\%). There was one transient common peroneal injury (Endoscope-assisted). There were no significant differences in proportion of intraoperative complications across procedure types for either Stenosis or Disc patients (Table 3a).

Postoperative complications occurred in 16 (4.7\%; 2 Endo, 11 Open, 3 Tube). No differences were observed in proportion of postoperative complications across procedure types for either Stenosis or Disc patients (Table 3a). In Open cases, one intraoperative dural tear became a persistent leak requiring reoperation, and a second patient developed a postoperative pulmonary embolism (Table $3 \mathrm{~b}$ ). In Tube-assisted group, one dural tear persisted as a CSF leak requiring reoperation, one patient developed meningitis (unrelated to index procedure) and another patient developed a urethral stricture. Endoscope-assisted patients experienced two wound infections.

\begin{tabular}{|c|c|c|c|c|c|}
\hline & \multirow[t]{2}{*}{ Total Cohort } & \multicolumn{2}{|c|}{ Stenosis Group $(n=104)$} & \multicolumn{2}{|c|}{ Disc Group ( $n=234)$} \\
\hline & & Frequency (\%) & p-value (method) & Frequency & p-value (method) \\
\hline Intra-Op Complications & $12(3.5 \%)$ & $8 / 104(7.7 \%)$ & & $4 / 234(1.7 \%)$ & \\
\hline Endo & 1 & -- & & $1 / 86(1.2 \%)$ & \\
\hline Open & 7 & $4 / 38(10.5 \%)$ & 0.46 & $3 / 100(3.0 \%)$ & 0.54 \\
\hline Tube & 4 & $4 / 66(6.1 \%)$ & & $0 / 48$ & \\
\hline Post-Op Complications & $16(4.7 \%)$ & 7/104 (6.7\%) & & $9 / 234(3.9 \%)$ & \\
\hline Endo & 2 & -- & & $2 / 86(2.3 \%)$ & \\
\hline Open & 11 & $5 / 38(13.2 \%)$ & 0.10 & $6 / 100(6.0 \%)$ & 0.45 \\
\hline Tube & 3 & $2 / 66(3.0 \%)$ & & $1 / 48(2.1 \%)$ & \\
\hline Discharged to SNF & $11(3.3 \%)$ & 8/104 (7.7\%) & & $3 / 234(1.3 \%)$ & \\
\hline Endo & 0 & -- & & $0 / 86$ & \\
\hline Open & 7 & 6/38 (15.8\%) & 0.05 & $1 / 100(1.0 \%)$ & 0.16 \\
\hline Tube & 4 & $2 / 66(3.0 \%)$ & & $2 / 48(4.2 \%)$ & \\
\hline
\end{tabular}

Table 3a: Complications \& Discharge Status.

\begin{tabular}{|c|c|c|c|c|}
\hline & n & Intraoperative & Postoperative & Discharge to SNF \\
\hline $\begin{array}{l}\text { Endoscopic } \\
\text { Assisted }\end{array}$ & 86 & $\begin{array}{l}1 \\
1 \text { - } \\
\text { palsy" }\end{array}$ & $\begin{array}{l}2 \\
1 \text { - Wound Infection } \\
1 \text {-Superficial Wound Infection }\end{array}$ & 0 \\
\hline Open & 138 & $\begin{array}{l}7 \\
7 \text { - Dural Tear++ }\end{array}$ & $\begin{array}{l}11 \\
1 \text { - Wound Dehiscence } \\
4 \text { - Wound Infection** } \\
2 \text { - lleus } \\
1 \text { - CSF Leak+ } \\
1 \text { - Pneumonia } \\
1 \text { - Pulmonary Embolism+ } \\
1 \text { - Cauda Equina }\end{array}$ & 7 \\
\hline Tube Assisted & 114 & 4 - Dural Tear+ & $\begin{array}{l}3 \\
1 \text { - CSF Leak+ } \\
1 \text { - Meningitis } \\
1 \text { - Urethral Stricture* }\end{array}$ & 4 \\
\hline
\end{tabular}

$+=$ Intraoperative occurrence associated with postoperative

* $=$ Postoperative occurrence associated with discharge to skilled nursing facility

Table 3b: Description of Complications. 
Eleven patients required discharge to skilled nursing facility $(3.3 \%$; 0 Endo, 7 Open, 4 Tube). There was a trend toward increased discharge to SNF among Stenotic patients undergoing Open procedures $(15.8 \%)$ compared to Tube $(3.0 \%, \mathrm{p}=0.05)$. No significant differences were observed for Disc group.

\section{Functional outcome measures (Table $4 a$ and $4 b$ )}

262 subjects were eligible for functional outcome measures at 10 months post-op (77.5\% of cohort). Among these, ten-month outcome data were captured for $110(42 \%)$.

Two-way ANOVA models conducted separately for Stenosis and Disc patient's interaction of procedure (Endo, Open Tube) by time (pre-op, 1 month, 4 months, 10 months) was not significant for any functional outcomes (ODI, VASL, or VASB). Overall effect of time was significant in all models, with post-hoc analyses indicating that functional outcomes improved over time regardless of procedure type or diagnostic group. Minimal clinically important differences (MCID) were evidenced by greater than 10 point reductions in mean ODI, 20 point reductions in mean VASB pain, and 30 point reductions in mean VASL from preop to 10 months post-op for all procedures (Tables $4 \mathrm{a}$ and $4 \mathrm{~b}$, Figures 1-6).

Economic impact (Table 5a and 5b: insurance providers, Table 6: Medicare LOS and ORT, Table 7a and 7b: cost, Table 8: reimbursement, Table 9 net revenue)

Insurance providers were classified as Private, Medicare, Medicaid, Worker's Compensation and uninsured. One hundred-seven patients (32.1\%) were Medicare beneficiaries.

Among Medicare beneficiaries, mean OR time for inpatients was greater for Open procedures as compared to Tube or Endoscopic (Table 6), for both Stenosis and Disc patients. Length of stay for Medicare inpatients was greatest in Tube procedure of the Disc group (5.6 days on average). Stenosis in patients undergoing Open procedures stayed in hospital for an average of 5.3 days.

Median costs per procedure were compared across procedure types

\begin{tabular}{|c|c|c|c|c|c|c|c|c|c|c|c|c|c|}
\hline & & & Preop & & & $1 \mathrm{Mo}$ & & & 4 Mo & & & $10 \mathrm{Mo}$ & \\
\hline \multirow[t]{2}{*}{ Procedure } & Test & $\mathbf{N}$ & Mean & Std & $\mathbf{N}$ & Mean & Std & $\mathbf{N}$ & Mean & Std & $\mathbf{N}$ & Mean & Std \\
\hline & ODI & 32 & 41.6 & 14.9 & 25 & 31.2 & 24.9 & 17 & 27.9 & 19.8 & 15 & 26.1 & 21.7 \\
\hline \multirow[t]{3}{*}{ Open } & VASB & 32 & 60.4 & 29.8 & 28 & 27.7 & 24.2 & 19 & 37.7 & 26.1 & 16 & 30.6 & 24.6 \\
\hline & VASL & 32 & 58.2 & 29.7 & 28 & 27.7 & 33.7 & 19 & 36.3 & 28.3 & 16 & 31.9 & 27.4 \\
\hline & ODI & 61 & 46.1 & 16.9 & 56 & 28.6 & 23.0 & 42 & 25.9 & 22.4 & 27 & 27.1 & 20.4 \\
\hline \multirow[t]{2}{*}{ Tube } & VASB & 62 & 60.7 & 28.9 & 57 & 26.7 & 27.1 & 45 & 28.4 & 30.7 & 27 & 27.8 & 27.1 \\
\hline & VASL & 61 & 59.9 & 31.2 & 57 & 25.3 & 29.6 & 45 & 27.8 & 31.9 & 27 & 30.4 & 34.1 \\
\hline
\end{tabular}

Table 4a: Functional Outcomes among Stenosis Group.

\begin{tabular}{|c|c|c|c|c|c|c|c|c|c|c|c|c|c|}
\hline & & & Preop & & & 1 Mo & & & 4 Mo & & & 10 Mo & \\
\hline \multirow[t]{2}{*}{ Procedure } & Test & $\mathrm{N}$ & Mean & Std & $\mathrm{N}$ & Mean & Std & $\mathrm{N}$ & Mean & Std & $\mathrm{N}$ & Mean & Std \\
\hline & ODI & 75 & 48.0 & 21.8 & 66 & 32.7 & 22.6 & 52 & 35.4 & 24.0 & 28 & 28.6 & 23.5 \\
\hline \multirow[t]{3}{*}{ Endo } & VASB & 77 & 67.4 & 27.8 & 69 & 36.9 & 26.5 & 52 & 42.7 & 29.2 & 31 & 39.4 & 29.5 \\
\hline & VASL & 76 & 69.3 & 27.6 & 69 & 37.1 & 29.8 & 53 & 38.7 & 31.6 & 30 & 36.0 & 28.5 \\
\hline & ODI & 83 & 54.1 & 17.1 & 83 & 31.5 & 21.1 & 53 & 29.2 & 21.8 & 21 & 33.1 & 25.6 \\
\hline \multirow[t]{3}{*}{ Open } & VASB & 81 & 63.0 & 31.9 & 85 & 35.2 & 28.1 & 52 & 34.7 & 29.9 & 20 & 44.4 & 34.3 \\
\hline & VASL & 80 & 72.5 & 26.7 & 81 & 33.6 & 30.3 & 48 & 36.3 & 32.0 & 20 & 35.9 & 32.6 \\
\hline & ODI & 43 & 48.8 & 16.0 & 33 & 30.8 & 25.1 & 23 & 29.9 & 23.3 & 16 & 24.6 & 25.8 \\
\hline \multirow[t]{2}{*}{ Tube } & VASB & 41 & 63.1 & 25.2 & 34 & 34.7 & 27.4 & 24 & 47.6 & 30.3 & 16 & 39.4 & 31.3 \\
\hline & VASL & 41 & 70.3 & 24.3 & 33 & 37.0 & 32.5 & 24 & 42.7 & 30.1 & 16 & 38.1 & 30.2 \\
\hline
\end{tabular}

Table 4b: Functional outcomes among Disc Group.

ODI - Stenosis Group

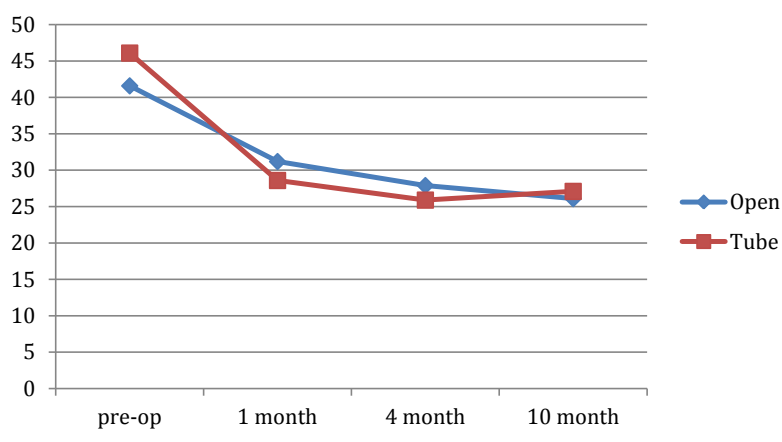

Figure 1: Stenosis Group Oswestry Disability Index. 


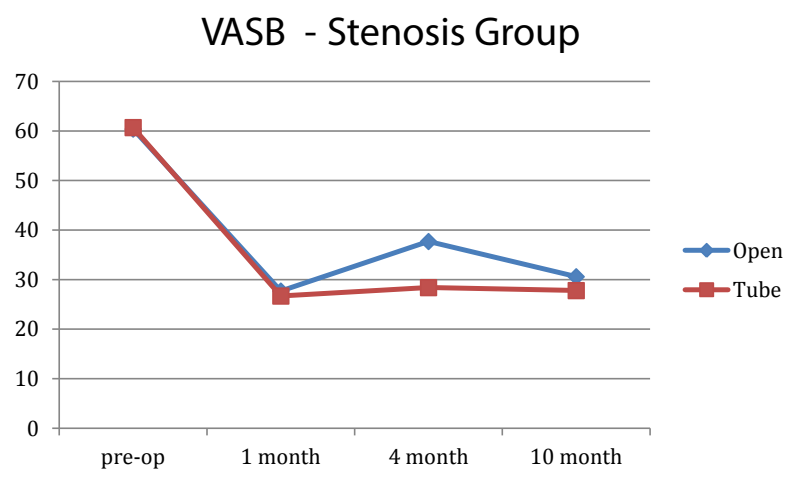

Figure 2: Stenosis Group Visual Analog Scale for Back Pain.

VASL - Stenosis Group

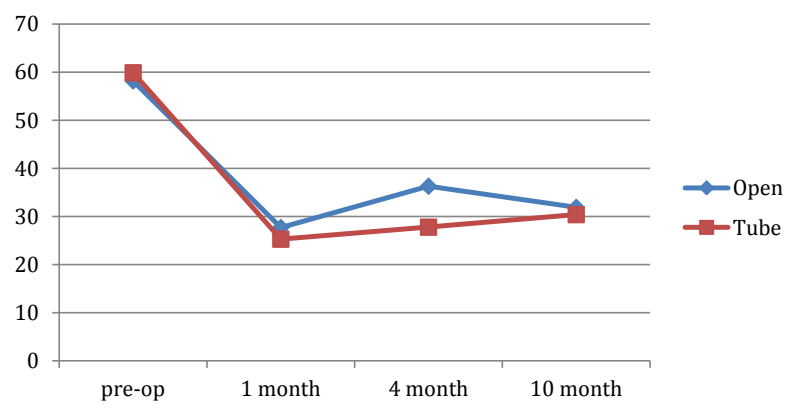

Figure 3: Stenosis Group Visual Analog Scale for Leg Pain.

\section{ODI - Disc Group}

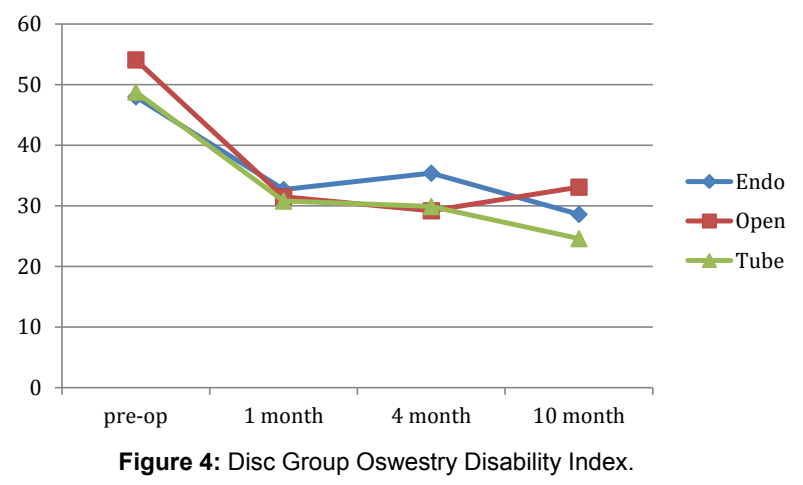

separately for Medicare-insured Stenosis and Disc patients. Among Stenosis patients (Table $7 \mathrm{c}$ ), median cost per procedure was significantly higher for Open procedures $(\$ 7,305)$ as compared to Tube $(\$ 4,518$, $\mathrm{p}<0.0001)$. There were no significant differences in cost by procedure (Endo vs. Open vs. Tube) for Disc patients $(\mathrm{p}=0.50$, Table $7 \mathrm{~d}$ ). Total costs, reimbursements, and revenue are presented for Medicare patients in Tables $7 \mathrm{a}, 7 \mathrm{~b}, 8 \mathrm{a}, 8 \mathrm{~b}, 9 \mathrm{a}$, and $9 \mathrm{~b}$.

\section{Discussion}

Our data demonstrate positive affect of direct lumbar decompression on functional outcome. Each procedure group reported minimal clinically important reductions in ODI, VASB and VASL regardless of pathology-spinal stenosis or disc excision. These findings are consistent with multiple centers $[4,7,10,11,13]$. Unlike Righesso and Shih $[6,10]$, our cohort did not experience an increase in operative time comparing less invasive procedures to Open cases. In fact, operative time for these procedures was less than Open. Surgical procedure and level of pathology were not bias towards lesser pathology being treated via less invasive techniques. The four surgeons supplying patients to this study used their standard criteria for patient selection and procedure. Two surgeons only performed the standard Open procedure. One surgeon with extensive experience using tubular retractors did not perform any open decompression during this study however would not exclude Open procedures from his practice if the indication presented itself. Lastly, this series does contain the "learning curve" and newly developed practice pattern of one surgeon committed to the use of endoscopes for single level pathology.

This report supports literature demonstrating a reduction in 


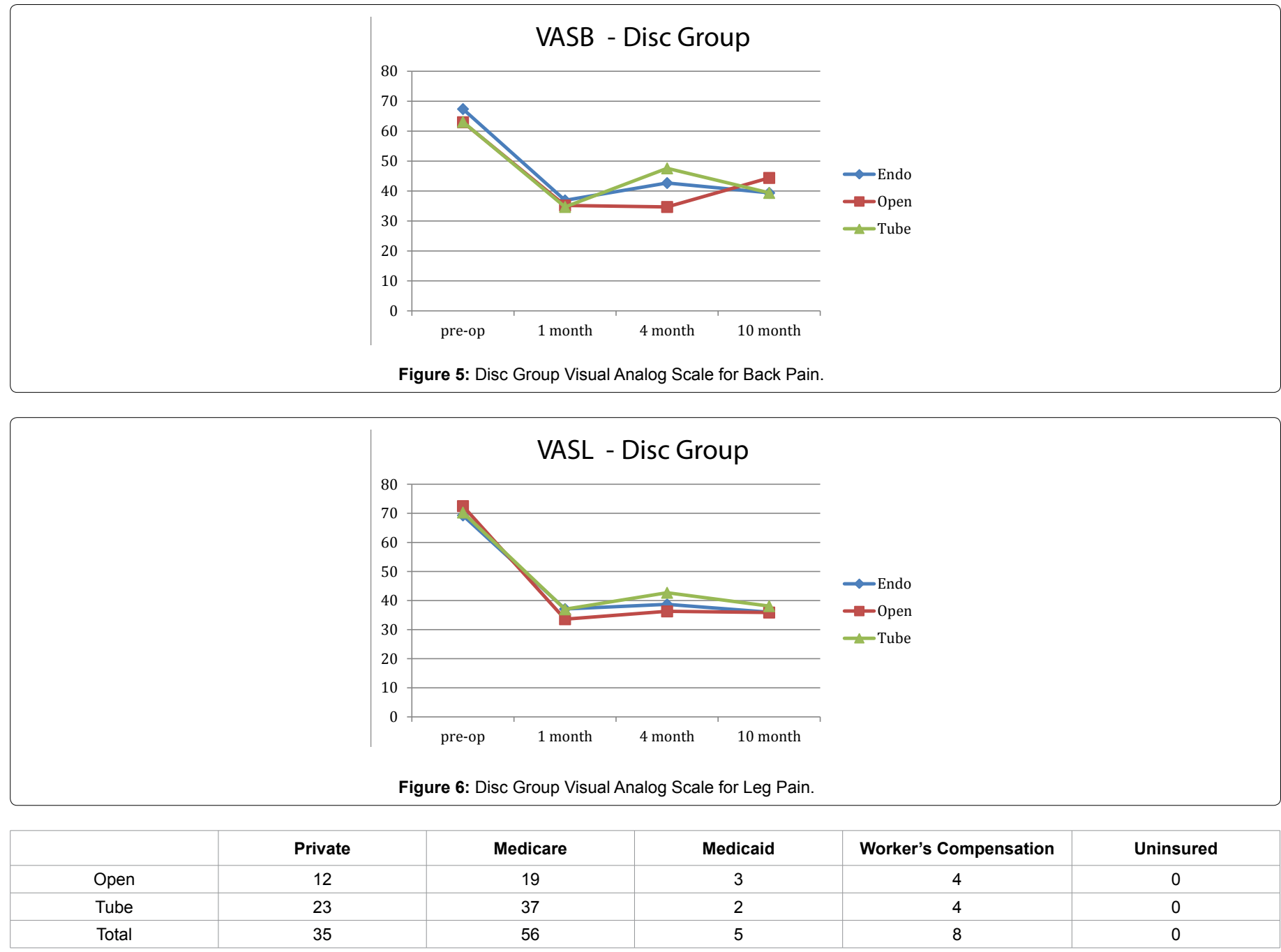

Table 5a: Insurance Providers - Stenosis Group $(n=104)$.

\begin{tabular}{|c|c|c|c|c|c|}
\hline & Private & Medicare & Medicaid & Worker's Compensation & Uninsured \\
\hline Endo & 40 & 23 & 12 & 8 & 3 \\
\hline Open & 66 & 13 & 9 & 10 & 2 \\
\hline Tube & 23 & 15 & 4 & 5 & 1 \\
\hline Total & 129 & 51 & 25 & 23 & 6 \\
\hline
\end{tabular}

Table 5b: Insurance providers - Disc Group ( $n=234)$.

\begin{tabular}{|l|c|c|c|}
\hline Procedure & Outpatient OR Time (minutes) & Inpatient OR Time (minutes) \\
\hline Stenosis Group $(n=56)$ & - & 224.5 \\
\hline Open & 108.0 & 135.0 \\
\hline Tube & & 5.3 \\
\hline & & 1.3 \\
\hline Disc Group $(n=51)$ & 69.5 & $64.0^{*}$ \\
\hline Endo & - & 148.2 \\
\hline Open & 83.7 & 94.9 \\
\hline Tube & & $1.0^{*}$ \\
\hline
\end{tabular}

${ }^{*} n=1$

Table 6: Medicare LOS \& ORT ( $n=107)$.

perioperative morbidity following less invasive procedure, but not increased patient reported back or leg pain [4]. Furthermore, patients undergoing Open procedures self-report a trend towards increased
ODI and VASB (Figures 1,2). 10-month sample size limits analysis of this trend. Standard deviations (Tables $4 \mathrm{a}$ and $4 \mathrm{~b}$ ) suggests right-sided shift in outcomes over time implying that each outcome measure, 
Citation: Knight RQ, Scribani M, Krupa N, Grainger S, Goldberg C, et al.(2013) Lumbar Decompressive Laminectomy or Laminotomy for Degenerative Conditions: "Outcome Comparison of Traditional Open versus Less Invasive Techniques". J Spine S2: 006. doi:10.4172/2165-7939.S2-006

Page 8 of 10

\begin{tabular}{|c|c|c|c|c|c|c|}
\hline Patient type and procedure & \# with Medicare & Mean OR Time & Cost per Minute & Mean LOS & Cost per day & Total Cost \\
\hline \multicolumn{7}{|l|}{ Outpatient } \\
\hline Open & - & & - & - & - & \\
\hline Tube & 11 & 108.0 & $\$ 22.16$ & - & - & $\$ 26,326.08$ \\
\hline \multicolumn{7}{|l|}{ Inpatient } \\
\hline Open & 19 & 224.5 & $\$ 22.16$ & 5.3 & $\$ 1,150$ & $\$ 209,534.56$ \\
\hline \multirow[t]{2}{*}{ Tube } & 26 & 135.0 & $\$ 22.16$ & 1.3 & $\$ 1,150$ & $\$ 116,881.60$ \\
\hline & & & & & & $\$ 326,416.16$ \\
\hline $\begin{array}{l}\text { GRAND TOTAL COST } \\
\text { Stenosis Group }\end{array}$ & & & & & & $\$ 352,742.24$ \\
\hline
\end{tabular}

Table 7a: Costs for Medicare Stenosis Group $(n=56)$.

\begin{tabular}{|c|c|c|c|c|c|c|}
\hline Patient type and procedure & \# with Medicare & Mean OR Time & Cost per Minute & Mean LOS & Cost per day & Total Cost \\
\hline \multicolumn{7}{|l|}{ Outpatient } \\
\hline Endo & 22 & 69.5 & $\$ 22.16$ & - & - & $\$ 33,882.64$ \\
\hline Open & - & - & - & - & - & - \\
\hline \multirow[t]{2}{*}{ Tube } & 7 & 83.7 & $\$ 22.16$ & - & - & $\$ 12,985.76$ \\
\hline & & & & & & $\$ 46,868.40$ \\
\hline \multicolumn{7}{|l|}{ Inpatient } \\
\hline Endo & 1 & 64.0 & $\$ 22.16$ & 1.0 & $\$ 1,150$ & $\$ 2,568.24$ \\
\hline Open & 13 & 148.2 & $\$ 22.16$ & 1.8 & $\$ 1,150$ & $\$ 69,130.16$ \\
\hline \multirow[t]{2}{*}{ Tube } & 8 & 94.9 & $\$ 22.16$ & 5.6 & $\$ 1,150$ & $\$ 68,569.44$ \\
\hline & & & & & & $\$ 140,267.84$ \\
\hline $\begin{array}{l}\text { GRAND TOTAL COST } \\
\text { Disc Group }\end{array}$ & & & & & & $\$ 187,136.24$ \\
\hline
\end{tabular}

Table 7b: Costs for Medicare Disc Group $(n=51)$.

\begin{tabular}{|c|c|c|c|c|c|c|}
\hline Procedure & \# Medicare Inpatient & Cost per Minute OR time & Median OR time cost & Cost per day Inpatient & Median LOS cost & Median Total Costs* \\
\hline Open & 19 & $\$ 22.16$ & $\$ 4,387.68$ & $\$ 1,150$ & $\$ 2,300.00$ & $\$ 7,305.84$ \\
\hline Tube & 26 & $\$ 22.16$ & $\$ 2,936.20$ & $\$ 1,150$ & $\$ 1,150.00$ & $\$ 4,518.32$ \\
\hline
\end{tabular}

${ }^{*} \mathrm{P}<0.0001$ by Wilcoxon rank sum test

Table 7c: Cost comparisons by procedure -Medicare Stenosis Group $(n=45)$.

\begin{tabular}{|c|c|c|c|c|c|c|}
\hline Procedure & \# Medicare Inpatient & Cost per Minute OR time & Median OR time cost & Cost per day Inpatient & Median LOS cost & Median Total Costs* \\
\hline Endo & 1 & $\$ 22.16$ & $\$ 1,418.24$ & $\$ 1,150$ & $\$ 1,150.00$ & $\$ 2,568.24$ \\
\hline Open & 13 & $\$ 22.16$ & $\$ 3,124.56$ & $\$ 1,150$ & $\$ 1,150.00$ & $\$ 4,895.04$ \\
\hline Tube & 8 & $\$ 22.16$ & $\$ 1,972.24$ & $\$ 1,150$ & $\$ 1,150.00$ & $\$ 4,451.84$ \\
\hline
\end{tabular}

${ }^{*} \mathrm{P}=0.50$ by Kruskal-Wallis test

Table 7d: Cost comparisons by procedure -Medicare Disc Group ( $n=22)$.

ODI, VASB or VASL, is associated with outliers continuing to report disabling symptoms at 10 months. Dynamics of patient reported ongoing life issues, which may blur appreciation of reported complaint, can confound functional outcome. Asghar and Hilibrand's review of SPORT data suggest that patients requiring lumbar decompression for spinal stenosis experience a significant reduction in symptoms by four months, which is sustained for 4 years [16]. Despite complex nature of patient reported outcomes data, Parker et al. has illustrated positive impact of traditional surgical decompression as a gain of $\mathrm{O} .72$ qualityadjusted-life-years postoperatively [11].

Today's medical economics have continued to pressure physician practices and healthcare systems to maximize return on resources. While "value" is the descriptive word of present choice, volume continues to drive financial stability. Migration from inpatient to ambulatory care has advanced within most subspecialties. Our data shows no significant differences in perioperative complication or functional outcomes based on location of service. Despite overall revenue positive aspects of lumbar decompression for degenerative conditions, open procedures were associated with highest cost to our organization (Table 7a). Medicare DRG and APC reimbursements produce a dramatic net revenue advantage for inpatient services. Recent enactment of Affordable Care Act emphasizes use of Recovery Audit Contractors (RACs) to recapture revenues for services provided to Medicare patients $[17,18]$. CMS guidelines, particularly regarding location of service, should be reviewed. Data here, based on "approximate accountings", fails to reflect true cost to our organization. Additional cost not reflected may contain a combination of professional (Anesthesia, Pathology, Primary Care, Radiology) and support (Dietary, Maintenance, Transport, etc.) services.

There are limitations in current study. Varied methodologies and definitions make it difficult to compare articles. Multiple functional outcome measures - SF-36, Oswestry, Roland-Morris, or VAS produce 
Citation: Knight RQ, Scribani M, Krupa N, Grainger S, Goldberg C, et al.(2013) Lumbar Decompressive Laminectomy or Laminotomy for Degenerative Conditions: "Outcome Comparison of Traditional Open versus Less Invasive Techniques". J Spine S2: 006. doi:10.4172/2165-7939.S2-006

Page 9 of 10

\begin{tabular}{|l|c|c|c|}
\hline Patient type and procedure & \# with Medicare & Reimbursement \\
\hline Outpatient & -- & \\
\hline Open & 11 & $\$ 4,518.00$ \\
\hline Tube & & $\$ 49,698.00$ \\
\hline & 19 & \\
\hline Inpatient & 26 & $\$ 16,837.00$ \\
\hline Open & & $\$ 16,837.00$ \\
\hline Tube & & $\$ 319,903.00$ \\
\hline \\
\hline $\begin{array}{l}\text { GRAND TOTAL REIMBURSEMENT } \\
\text { Stenosis Group }\end{array}$
\end{tabular}

Table 8a: Reimbursements - Medicare Stenosis Group $(n=56)$

\begin{tabular}{|c|c|c|c|}
\hline Patient type and procedure & \# with Medicare & Reimbursement & Total Reimbursement \\
\hline \multicolumn{4}{|l|}{ Outpatient } \\
\hline Endo & 22 & $\$ 4,518.00$ & $\$ 99,396.00$ \\
\hline Open & - & $\$ 4,518.00$ & - \\
\hline \multirow[t]{2}{*}{ Tube } & 7 & $\$ 4,518.00$ & $\$ 31,626.00$ \\
\hline & & & $\$ 131,022.00$ \\
\hline \multicolumn{4}{|l|}{ Inpatient } \\
\hline Endo & 1 & $\$ 16,837.00$ & $\$ 16,837.00$ \\
\hline Open & 13 & $\$ 16,837.00$ & $\$ 218,881.00$ \\
\hline \multirow[t]{2}{*}{ Tube } & 8 & $\$ 16,837.00$ & $\$ 134,696.00$ \\
\hline & & & $\$ 370,414.00$ \\
\hline \multicolumn{3}{|c|}{ GRAND TOTAL REIMBURSEMENT Disc Group } & $\$ 501,436.00$ \\
\hline
\end{tabular}

Table 8b: Reimbursements - Medicare Disc Group $(n=51)$.

\begin{tabular}{|c|c|c|c|c|c|}
\hline Procedure & Net Outpatient Revenue & Net Inpatient Revenue & $\%$ Net Outpatient Revenue & $\%$ Net Inpatient Revenue & Total Medicare Revenue \\
\hline Open & & $\$ 110,368.44$ & & 34.5 & $\$ 110,368.44$ \\
\hline Tube & $\$ 23,371.92$ & $\$ 320,880.40$ & 47.0 & 73.3 & $\$ 344,252.32$ \\
\hline
\end{tabular}

Table 9a: Net revenues among Medicare Stenosis Group $(n=56)$.

\begin{tabular}{|c|c|c|c|c|c|}
\hline Procedure & Net Outpatient Revenue & Net Inpatient Revenue & $\%$ Net Outpatient Revenue & \% Net Inpatient Revenue & Total Medicare Revenue \\
\hline Endo & $\$ 65,513.36$ & $\$ 14,268.76$ & 65.9 & 84.7 & $\$ 79,782.12$ \\
\hline Open & & $\$ 149,750.84$ & & 68.4 & $\$ 149,750.84$ \\
\hline Tube & $\$ 18,640.24$ & $\$ 66,126.56$ & 58.9 & 49.1 & $\$ 84,766.80$ \\
\hline
\end{tabular}

Table 9b: Net revenues among Medicare Disc Group $(n=51)$.

data that is comparable but not identical. We chose disease specific measures (Oswestry, VAS) and lack general instruments, such as Short Forms (36, 24, or 12). Complex statistics make interpretation and understanding of data demanding. Defining actual procedure studied can also present difficulty. Initial reports of microendoscopic procedures actually represent early development of tubular retractors [3].

Registry data can be subject to bias. Our data was collected prospectively and reviewed retrospectively. Patient selection criteria and patient or physician compliance with data completion can bias registry information by reducing available data for analysis. McGirt et al. established a registry with data points of preop, three and twelve months hoping for a high level (80\%) follow-up [19]. Our registry is ongoing leaving true follow-up percentage yet to be determined. Reliance upon clerical staff for data entry cannot overcome failure of physicians or patients to comply with data submission and completion. Armstrong et al. reported on impact of HIPAA consenting resulting in reduced compliance percentages ranging from $96 \%$ to $34 \%$ [20]. Fortytwo percent of our initial enrollment completed sufficient data points at
10 months. Distance traveled to follow-up, its economic impact in this rural setting, lack of patient compensation and physician commitment to data completion are all mitigating factors resulting in reduced followup. Patients lost to follow-up are typically considered poor outcomes in intent-to-treat analysis of clinical trials. Registry data is a form of longitudinal study and may still provide valuable information at less than $80 \%$ follow-up.

In summary, spinal decompression for lumbar degenerative conditions is an excellent procedure performed via open or minimally invasive technique (Endoscope-assisted or Tube-assisted). Information supplied here documents overall effectiveness of lumbar decompression for degenerative conditions. Improved patient functional outcome should be expected regardless of technique chosen. Decisions to treat lumbar conditions and where those services are provided have an economic impact on not only surgeons but also their hospital or healthcare system. While revenue considerations favor delivery of services as in-patients, single level decompression for patients without significant co-morbidities is clearly amenable to outpatient treatment. 
Surgeon choices between open versus minimally invasive techniques may be somewhat generational. Patients and other consumers of healthcare services continue to inform themselves and participate as partners in this complex decision-making process.

\section{Disclaimer}

The arguments and conclusions that the authors express in this manuscript are entirely theirs and do not represent the official views of any institutions to which the authors are affiliated.

\section{References}

1. Khoo LT, Fessler RG (2002) Microendoscopic decompressive laminotomy for the treatment of lumbar stenosis. Neurosurgery 51: S146-154.

2. Caspar W (1977) A new surgical procedure for lumbar disk Herniation causing less tissue damage through a microsurgical approach. Adv Neurosurg 4: 74-77.

3. Foley KT, Smith MM (1997) Microendoscopic discectomy. Tech Neurosurg 3: 301-307.

4. Arts MP, Brand R, van den Akker ME, Koes BW, Bartels RH, et al. (2009) Tubular diskectomy vs conventional microdiskectomy for sciatica: a randomized controlled trial. JAMA 302: 149-158.

5. Hermantin FU, Peters T, Quartararo L, Kambin P (1999) A prospective, randomized study comparing the results of open discectomy with those of video-assisted arthroscopic microdiscectomy. J Bone Joint Surg Am 81: 958965.

6. Shih P, Wong AP, Smith TR, Lee Al, Fessler RG (2011) Complications of open compared to minimally invasive lumbar spine decompression. J Clin Neurosci 18: $1360-1364$

7. Wu X, Zhuang S, Mao Z, Chen H (2006) Microendoscopic discectomy for lumbar disc herniation: surgical technique and outcome in 873 consecutive cases. Spine (Phila Pa 1976) 31: 2689-2694.

8. German JW, Adamo MA, Hoppenot RG, Blossom JH, Nagle HA (2008) Perioperative results following lumbar discectomy: comparison of minimally invasive discectomy and standard microdiscectomy. Neurosurg Focus 25: E20.
9. Harrington JF, French P (2008) Open versus minimally invasive lumbar microdiscectomy: comparison of operative times, length of hospital stay, narcotic use and complications. Minim Invasive Neurosurg 51: 30-35.

10. Righesso O, Falavigna A, Avanzi O (2007) Comparison of open discectomy with microendoscopic discectomy in lumbar disc herniations: results of a randomized controlled trial. Neurosurgery 61: 545-549.

11. Parker SL, Adogwa O, Davis BJ, Fulchiero E, Aaronson O, et al. (2011) Costutility Analysis of Minimally Invasive Versus Open Multilevel Hemilaminectomy for Lumbar Stenosis. J Spinal Disord Tech 26: 42-47.

12. Parker SL, Fulchiero EC, Davis BJ, Adogwa O, Aaronson OS, et al. (2011) Costeffectiveness of multilevel hemilaminectomy for lumbar stenosis-associated radiculopathy. Spine J 11: 705-711.

13. Ryang YM, Oertel MF, Mayfrank L, Gilsbach JM, Rohde V (2008) Standard open microdiscectomy versus minimal access trocar microdiscectomy: results of a prospective randomized study. Neurosurgery 62: 174-181.

14. Goldfield N (2010) The evolution of diagnosis-related groups (DRGs): from its beginnings in case-mix and resource use theory, to its implementation for payment and now for its current utilization for quality within and outside the hospital. Qual Manag Health Care 19: 3-16.

15. Konstantinou K, Dunn KM (2008) Sciatica: review of epidemiological studies and prevalence estimates. Spine (Phila Pa 1976) 33: 2464-2472.

16. Asghar FA, Hilibrand AS (2012) The impact of the Spine Patient Outcomes Research Trial (SPORT) results on orthopaedic practice. J Am Acad Orthop Surg 20: 160-166.

17. Goldberg Aj, Young LM (2011) What every CEO should know about Medicare's recovery audit contractor program. J Healthc Manag 56: 157-161.

18. https://www.fbo.gov/index?s=opportunity\&mode=list\&tab=list\&_nfound=1

19. McGirt MJ, Speroff T, Dittus RS, Harrell FE, Asher AL (2013) The National Neurosurgery Quality and Outcomes Database (N2QOD): general overview and pilot-year project description. Neurosurgery Focus 34: E6.

20. Armstrong D, Kline-Rogers E, Jani SM, Goldman EB, Fang J, et al. (2005) Potential impact of the HIPAA privacy rule on data collection in a registry of patients with acute coronary syndrome. Arch Intern Med 165: 1125-1129. 